\title{
FEMINIZACIÓN DE LA UNIVERSIDAD DE GRANADA EN EL ÚLTIMO CUARTO DEL SIGLO XX: EL ACCESO DEL PROFESORADO
}

\author{
Ana María Muñoz-Muñoz \\ Universidad de Granada
}

\section{Introducción}

En 1975 la formación del movimiento de mujeres se acelera, las Naciones Unidas celebran el Año Internacional de la Mujer dando lugar en España a encuentros como las “Primeras Jornadas por la Liberación de la Mujer" (Madrid, 6 al 8 de diciembre de 1975) y las "Primeres Jornades Catalanes de la Dona" (Barcelona, 27 al 30 de mayo de 1976). Se proclama la igualdad de acceso a las enseñanzas para varones y mujeres, pero las nuevas generaciones, lo mismo que las anteriores, se orientan hacia áreas de conocimiento feminizadas. En las universidades las ramas preferidas siguen siendo las de Filosofía y Letras, Filología, Farmacia y, en menor medida, las de Medicina.

El período de la transición a la democracia supone para España en materia de investigación un paso importante en el sistema científico y tecnológico como la creación del Ministerio de Universidades e Investigación el 6 de abril de 1979, en el gobierno de UCD (Unión de Centro Democrático), y posteriormente las reformas del programa electoral del PSOE (Partido Socialista Obrero Español) que anunciaba una "Ley de Autonomía Universitaria” así como más recursos para $\mathrm{I}+\mathrm{D}$, además de la promulgación de 
la Ley de Reforma Universitaria (L.R.U.) del 25 de agosto de $1983^{1}$ y de la Ley de Fomento y Coordinación General de la Investigación Científica y Técnica aprobada el 14 de abril de 1986.

Con estos cambios políticos y sociales la Universidad se convierte en un espacio democrático, como lugar de reunión, de intercambio y debate. Desde las aulas se lucha contra la dictadura; a través de manifestaciones se expresan y defienden los nuevos ideales de hombres y mujeres. El trabajo científico es resultado y fruto de la persona que investiga, de su visión del mundo, de su posición social, por ello la producción científica durante este período nos va a permitir ver los intereses y metas de las profesoras universitarias en Granada.

\subsection{El acceso a las mujeres a las universidades españolas: breve contexto histórico}

Desde mediados del siglo XIX, comienza un proceso lento y difícil de incorporación de la mujer a las universidades españolas cuyos hitos principales vamos a recorrer a continuación. Siguiendo a Consuelo Flecha (1996), las primeras mujeres que acceden a la Universidad no debemos considerarlas como casos aislados, sino como pioneras en la integración de la mujer en la enseñanza superior. Nos encontramos en un período de la historia de España en el que las condiciones socioculturales propician que sea el hombre el que decida sobre lo que desean las mujeres, nuestros problemas eran planteados por los varones excluyendo y ocultando nuestras opiniones, con el temor de ser desplazados y perder la autoridad que les estaba confiada, reafirmando a su vez, el poder del patriarcado. Sus estrategias son de las más diversas y humillantes en cuanto a

${ }^{1}$ Ley que se constituyó con el régimen democrático socialista en 1983. La L.R.U fue un instrumento de transformación social, al servicio de la libertad, la igualdad y el progreso social, que abogaba por el principio de igualdad y por eliminar la estructura jerárquica del profesorado. Por ejemplo con respecto a la selección y promoción del profesorado hace referencia al respeto de los principios de méritos, publicidad y no-discriminación que debe regir la asignación de todo puesto de trabajo por parte del Estado español. 
Feminización de la Universidad de Granada...

nivel de inteligencia del sexo femenino y, como defendía racionalmente Concepción Arenal (1823-1893): "Ni el estudio de la fisiología del cerebro ni la observación de lo que pasa en el mundo autorizan para afirmar resueltamente que la inferioridad intelectual de la mujer sea orgánica, porque no existe en las clases educadas, sino donde empieza la diferencia de la educación" (Arenal,1989: 20). El deseo de las mujeres a acceder a una educación superior, hacía cambiar valores sociales que muchos no admitían.

En 1868, cuando Isabel II es derrocada por los liberales, el sistema político social hace cambiar la legislación y en especial la educativa, se proclama la libertad de enseñanza, la ley no mencionaba a las jóvenes pero tampoco las excluía. La primera mujer admitida en la universidad fue matriculada en la Facultad de Medicina de Barcelona, en el curso académico 1872-73, pocos meses antes de proclamarse la I República; para ello tuvieron que pedir un permiso especial al rey Amadeo de Saboya, que se manifestó en una Real Orden del 2 de septiembre de 1871. En el mismo año, por Orden de la Dirección General de Instrucción Pública se concedía a dos jóvenes realizar el examen de ingreso de segunda enseñanza, pero en régimen privado, no oficial, por los inconvenientes de compartir aulas con los chicos. Las pioneras universitarias eran mujeres notables y singulares, procedentes de clases sociales altas, que iban a transmitir, por su forma de actuar, nuevos modos de vida a otras mujeres. Debemos recordar que no todas tenían la oportunidad de estudiar ya que el índice de analfabetismo era muy elevado en la población española: en 1860 para las mujeres era de un $90.9 \%$ sobre la población total y para los hombres de un 68.9\%; a finales del XIX el índice para las mujeres es de un $74.4 \%$ y para los hombres de un $57.8 \%$. Apenas sin darse cuenta, estas mujeres reclamaban el derecho a estar presentes en espacios públicos que hasta ese momento habían sido creados por y para hombres. Su acceso a la universidad fue gradual, y todo gracias al tesón e insistencia de estas mujeres ante las autoridades ministeriales de esos momentos.

El período de la Restauración, finales de 1874, trae consigo una nueva etapa política. En esta época se expiden títulos de Bachiller, pero para las mujeres no tenían 
efecto profesional, sólo académico. A las mujeres no se les prohibía estudiar, se las animaban a obtener el título para elevar su nivel intelectual. Algunas de ellas que obtienen el título de Bachiller desean entrar en la Universidad, situación que no habían considerado los dirigentes de la época. En 1882 una Real Orden permite acceder al Grado y al Título de Medicina a dos mujeres, pero sin que sirviera de antecedente a otras. Ambas mujeres, una vez terminados sus estudios en 1878, y antes de esta Real Orden solicitan su título al Consejo de Instrucción Pública, tardando tres años en elaborar un dictamen, para que el Ministerio acordase si concederle o no el título a las dos. En Enero de 1882 se les expedían sus títulos correspondientes y se les autorizaba el ejercicio de su profesión. Asimismo se hicieron varias propuestas relativas a las consecuencias que iban a suponer el reconocimiento de que estas mujeres finalizasen sus estudios; tales como si esta concesión iba a trascender a otras que estuviesen en la misma situación y si estos títulos iban a permitir ejercer la profesión, por último sobre el modo de organización de la educación de las mujeres se sugería crear un plan de estudios específicos para ellas. Estas propuestas fueron votadas, quedando aprobado expedir el título académico tanto a las solicitantes como a todas aquéllas que estuviesen matriculadas en la actualidad. Consecuentemente se trató el punto referido al ejercicio de la profesión de Medicina por mujeres, privando a estas de acceder a ningún puesto de la administración pública, y sobre el tipo de centro en la que podrían hacer sus estudios. El dictamen concluyó con facilitar estos estudios a las mujeres y procurar reformas legislativas que constituyeran un plan de enseñanza de la mujer atendiendo a las condiciones de su sexo.

En 1882 el Vizconde de Campo-Grande, Diputado en las Cortes y Senador del Reino, expresaba que socialmente existían dos problemas: el derecho de la mujer a la instrucción y el derecho de la misma al trabajo. Para solventar el primer problema se sigue como ejemplo la medida tomada por el Instituto de Valencia, en el que se admite la matrícula y examen de mujeres siempre que estudien privadamente y no asistan públicamente a centros y aulas de docencia, hasta que no fueran creadas instituciones regentadas por mujeres, como ocurría en los países anglosajones, y que no llegaron a implantarse en España. Las concesiones se 
Feminización de la Universidad de Granada...

otorgaban provisionalmente, cada vez que se presentaba una solicitud, y siempre tenían algún límite, primero, el de ejercer la profesión y segundo, poder cursar la Segunda Enseñanza sin que les diese el derecho a cursar estudios en las Universidades.

En el último decenio del XIX hay una mayor preocupación por la educación y por el acceso de la mujer a las universidades. En 1892 se celebra en Madrid el Congreso Pedagógico Hispano-Portugués, una de las secciones se dedicó a la enseñanza de la mujer, a la que asistieron entre otras Concepción Arenal y Emilia Pardo Bazán, se reivindicó el derecho de las mujeres a recibir la misma educación que el hombre y a acceder a todos los grados de educación, así como a ejercer la Medicina y la Farmacia; no obstante se les niega ejercer su profesión, y la coeducación se reconoce sólo en las enseñanzas primarias.

Una vez obtenido el reconocimiento legal, el siglo XIX va a traer cambios que favorecerán el acceso a las mujeres en la universidad. En 1910 se crea una Real Orden que permite a cualquier mujer que desee matricularse en la enseñanza universitaria pueda hacerlo sin consulta previa a los órganos de gobierno, pudiendo ejercer profesionalmente con el Título académico.

En el curso 1900/10 había 21 alumnas matriculadas en las universidades españolas (Madrid, Barcelona, Salamanca y Valencia): doce en Medicina, tres en Farmacia, tres en Ciencias, dos en Derecho y una en Filosofía. Representando un $0.1 \%$ del total del alumnado, que aumentará un $0.2 \%$ en las carreras médicas y farmacéuticas y de ciencias, siendo muy bajos en Filosofía (0.06\%) y Derecho (0.04\%) (Capel, 1986: 462-63).

Para el curso 1919-1920, las mujeres representaban un 2\%, y desde los años veinte a los cuarenta un $13.3 \%$. Aquellas mujeres que terminan sus estudios con éxito comienzan a ejercer profesionalmente, entre las primeras que desempeñan profesiones liberales hay cirujanas, médicas rurales, y arquitectas (Campo, 1963: 228-230). En el curso 1919/20 la Universidad de Granada cuenta con 24 alumnas y para el curso 1927/28 con 124, siendo la tercera universidad con más número de mujeres matriculadas después de Madrid y Barcelona. Las Facultades con mayor número de alumnas son las de Farmacia, Filosofía y Letras y Ciencias, por ser las que sus estudios ofrecían salidas profesionales. Las menos solicitadas eran Medicina por su carácter limitado en las ramas de Pediatría y 
Ginecología, y además por la aparición de nuevos campos laborales entre ellos la enseñanza media y superior y el acceso al cuerpo de Archiveros, Bibliotecarios y Arqueólogos trabajos que requerían el título de Filosofía y Letras. Por último, la Facultad de Derecho por ser la que menos posibilidades de trabajo ofrecía y por estar prohibidas a las mujeres oposiciones como las de Judicatura, Abogacía del Estado, Notaría, etc. (Capel, 1986: 468-71).

Con la creación de las Escuelas Normales de Maestras a principios del siglo XX comienza una progresiva presencia de la mujer en el estamento docente, pero no es hasta finales de los años veinte cuando las mujeres acceden al cuerpo de Catedráticos de Instituto siendo requisito imprescindible tener un título universitario.

Es a partir de 1930 cuando algunas mujeres van a estar presentes en la universidad como auxiliares en facultades como la de Ciencias en Zaragoza, Física y Pedagogía en la Universidad Central y Filosofía y Letras en Madrid (Capel, 1986: 496-99). En la Universidad de Granada las primeras mujeres docentes pertenecieron a la Facultad de Filosofía y Letras, éstas fueron Dña. Joaquina Eguaras Ibáñez que ejerce como ayudante de clases prácticas desde 1925 y Dña. Julia Rodríguez Danielovsky que ocupó el puesto de auxiliar temporal en 1930 (Torres, 2001: 183-4). En los años cuarenta, en España, no había ninguna profesora de Escuelas Técnicas Superiores (ETS) y en las Facultades el porcentaje era sólo del 4.5\%; en 1960 la situación no había variado en cuanto a ETS y eran mujeres el $8.5 \%$ del profesorado de Facultades.

Una vez realizado el repaso de las dificultades que tuvieron que ir sorteando las mujeres para acceder al mundo universitario, me propongo analizar la incorporación de profesoras a la Universidad de Granada. Este interés radica en percibir aspectos relativos a su distribución en función de distintas variables de corte académico-profesional (tipo de área de conocimiento en la que trabaja, de categoría a la que pertenece y de años en esta categoría) con el fin de establecer conexiones entre estas variables y las distintas situaciones de presencia/ausencia de profesoras. Es decir, analizar la información de los parámetros básicos de la situación de las profesoras en la estructura de la Universidad de Granada. 


\section{Fuentes y metodología}

Las fuentes consultadas han sido facilitadas por el Vicerrectorado de Ordenación Académica de la Universidad de Granada, engloba tres tipos de datos: número de profesorado por años que fueron contratados en la Universidad de 1975 a 1990, áreas de enseñanza a las que pertenecen agrupadas según la clasificación del Ministerio de Educación y Ciencia en tres grandes áreas de conocimiento: Ciencias Humanas y Sociales (CHS), Ciencia y Tecnología (CT) y Ciencias Médicas y de la Vida (CMV) y categorías académicas a las que pertenecen a lo largo del período; esta última categoría debido a la gran diversidad y a los cambios que sufren en su denominación hemos optado por clasificarlas en dos grupos: Funcionarias y Profesorado No Numerariros (PNNs).

Una vez consultadas las fuentes procedimos a la recopilación, selección, organización y ordenación de los datos, para posteriormente aplicar el análisis cuantitativo descriptivo de los mismos. Los datos académicos del profesorado han sido tabulados y organizados en una base de datos creada con el programa Access y posteriormente, los datos se representaron gráficamente en la hoja de cálculo Excel.

El método utilizado ha sido el análisis cuantitativo-descriptivo aplicado a los siguientes aspectos: la incorporación de las profesoras a la Universidad de Granada.

- El proceso de incorporación de las profesoras a la Universidad de Granada lo analizamos utilizando los siguientes indicadores:

- $\quad$ Número y porcentaje de profesorado por años y sexo.

- Porcentaje de mujeres en el profesorado universitario en CHS, en CT y en CMV por años. 
- $\quad$ Tasa anual de crecimiento del profesorado femenino en CHS, en CT y en CMV

- Porcentaje de mujeres en el profesorado universitario por años, teniendo en cuenta el número de áreas de conocimiento específicas pertenecientes a CHS, CT y CMV.

- Para analizar los cambios de categoría académica desde el año 1975 hasta 1990, hemos creído conveniente dividirlas en grupos según el tipo de contratación, ya que durante este período existe una gran variedad categorías. Para su clasificación hemos tomado como referencia el grado de doctor. La incorporación de las profesoras en las distintas categorías y la variación de las mismas en las distintas áreas de conocimiento las analizamos utilizando los siguientes indicadores:

- $\quad$ Porcentaje de funcionarias/os y de PNNs / Asociados por sexo.

- $\quad$ Porcentaje de funcionarias/os y de PNNs / Asociados por sexo en CHS, en CT y en CMV.

\section{Resultados}

\subsection{Incorporación a la academia: distribución por años}

En los inicios de la incorporación de las mujeres al mercado laboral, años setenta, se aprueba la Ley General de Educación (1970), pretendiendo la igualdad de oportunidades educativas, sin diferenciar asignaturas específicas según sexo. Inés Alberdi e Isabel Alberdi (1984:10) observan que la Ley no termina por abolir las discriminaciones en razón de sexo, ya que no se hacían planteamientos explícitos sobre la manera de potenciar una igualdad de oportunidades educativas que fuese real. Podemos decir que hasta tiempos recientes, la educación fue más un privilegio de pocos que derecho de todos. Además, esta Ley abrió las puertas a la incorporación de la enseñanza superior no universitaria a la universidad. Así, las ETS de ingeniería y de arquitectura, los estudios de administración de empresas, de magisterio, de enfermería, entre otros, pasaron a formar parte del sistema tradicional de universidad. 
Feminización de la Universidad de Granada...

La creciente presencia de las mujeres en la universidad española a partir de los años sesenta hay que explicarla en el conjunto del fenómeno de masificación que tiene lugar por el acceso de los sectores sociales más bajos. Desde el año 1940 las mujeres pasaron a representar de un $13 \%$ hasta un $37 \%$ en 1975 , alcanzando un $50 \%$ o más en la actualidad. Esta feminización del alumnado se ha ido traduciendo en una progresiva, aunque lenta, incorporación de las mujeres al profesorado siempre en las escalas de menor categoría (DURAN, 1981).

En la Universidad de Granada, el número de profesoras a comienzos del período de estudio es escaso, pero también lo era el conjunto de todo el profesorado. Así lo demuestran los datos del Vicerrectorado de Ordenación Académica de la Universidad de Granada que hemos utilizado, aunque las cifras en números absolutos varían si las comparamos con los datos que ofrecen el Consejo de Universidades a partir de 1988 y el Instituto Nacional de Estadística (I.N.E.) la evolución en cifras porcentuales varía muy poco. No obstante, para tener un mejor conocimiento de los fenómenos, utilizaremos ambos datos para observar comparativamente la evolución del profesorado de la Universidad de Granada con el conjunto de las universidades españolas.

Conforme va creciendo el personal docente en la universidad, va aumentando el número de profesoras hasta las 784 que hay en 1990. En ese proceso de aumento paulatino del profesorado, nos interesa observar qué proporción han supuesto las mujeres dentro de ese colectivo. Si en los últimos cinco años de la década de los setenta no llegaban a representar el $20 \%$, en la primera mitad de la década siguiente superan ese porcentaje, llegando a haber aproximadamente una mujer por cada tres varones en 1985 . En el quinquenio final de la década, la proporción de mujeres con respecto al total del profesorado aumenta escasamente, sin llegar si quiera a representar un $30 \%$ del profesorado. En consecuencia, si desde 1975 a 1990 el profesorado femenino aumenta como también lo hace la propia Universidad y el personal docente, el avance que consigue en cuanto a representación y en comparación con los varones es modesto, ya que si en 1990 las mujeres suponen aproximadamente un 10\% más de lo que suponían 
en 1975, escasamente superan la cuarta parte del total del profesorado en el último año del período estudiado (ver tab. 1).

Tabla 1

Evolución anual del profesorado en la Universidad de Granada (1975-90)

\begin{tabular}{lllllc}
\hline & \multicolumn{2}{c}{ Mujeres } & \multicolumn{2}{c}{ Hombres } & Ambos-sexos \\
\hline 1975 & 27 & $18.7 \%$ & 117 & $81.2 \%$ & 144 \\
1976 & 33 & $18.7 \%$ & 143 & $81.2 \%$ & 176 \\
1977 & 34 & $17.7 \%$ & 158 & $82.3 \%$ & 192 \\
1978 & 43 & $18.4 \%$ & 191 & $81.6 \%$ & 234 \\
1979 & 53 & $19.9 \%$ & 213 & $80.1 \%$ & 266 \\
1980 & 60 & $20.2 \%$ & 237 & $79.8 \%$ & 297 \\
1981 & 75 & $21.2 \%$ & 279 & $78.8 \%$ & 354 \\
1982 & 86 & $22.2 \%$ & 301 & $77.8 \%$ & 387 \\
1983 & 105 & $20.5 \%$ & 406 & $79.5 \%$ & 511 \\
1984 & 133 & $21.2 \%$ & 495 & $78.8 \%$ & 628 \\
1985 & 215 & $25.7 \%$ & 622 & $74.3 \%$ & 837 \\
1986 & 253 & $24.9 \%$ & 765 & $75.1 \%$ & 1.018 \\
1987 & 386 & $27.0 \%$ & 1.041 & $73.0 \%$ & 1.427 \\
1988 & 495 & $28.0 \%$ & 1.272 & $72.0 \%$ & 1.767 \\
1989 & 654 & $28.3 \%$ & 1.654 & $71.7 \%$ & 2.308 \\
1990 & 784 & $28.0 \%$ & 2.021 & $72.0 \%$ & 2.805 \\
\hline
\end{tabular}

El aumento del profesorado es continuo durante todo el período, pero desigual para mujeres y varones. De los 2.661 docentes que se incorporan a la Universidad de Granada, apenas un $28.4 \%$ corresponde a mujeres, lo cual contribuye a mantener esa estructura desigual en cuanto a presencia de mujeres en el profesorado universitario.

El aumento anual del profesorado femenino en la Universidad de Granada fue escaso hasta mediados de los ochenta, por lo que no podemos hablar de un incremento constante sino irregular hasta 1985. En valores absolutos para el año 1975 había un total de 27 profesoras que representaban un $18.7 \%$ del total del profesorado. Las mejoras de la L.R.U. no se perciben hasta el año 1985 en el que son contratadas 82 profesoras lo que supuso casi el $40 \%$ del incremento total del personal docente en ese año. El 
Feminización de la Universidad de Granada...

incremento anual de profesoras supone una presencia un $10 \%$ mayor de mujeres en 1990, pero para que en ese mismo año el número de profesores varones suponga todavía más del $70 \%$, su incremento anual en números absolutos ha tenido que ser bastante más elevado que el de mujeres.

Como hemos visto, la mayor presencia de mujeres en el profesorado durante el período estudiado es todavía insuficiente y muy desigual con respecto al profesorado masculino, pero bien pudiera estar anunciando mejoras considerables para años posteriores, mejoras que de ninguna manera supondrán una estructura de presencia igualitaria de ambos sexos, sin la toma de conciencia de la situación de las mujeres.

Tanto en las universidades españolas como en la Universidad de Granada ${ }^{2}$ el profesorado femenino ha sobretriplicado sus efectivos, pasando de 5.449 profesoras en 1975 a 18.644 en 1990 y de 239 en 1975 a 892 en 1990, respectivamente. En relación con el conjunto del profesorado el aumento ha sido de un $10.1 \%$ para las universidades españolas y de un $8.3 \%$ para la Universidad de Granada, pudiéndose afirmar que se da un avance importante en el proceso de tender hacia un mayor equilibrio entre los dos sexos dentro del profesorado. En 1990 el porcentaje de profesoras, en ambos casos, es aproximadamente de un $30 \%$, todavía lejos de la paridad que le correspondería en tanto que representan el $50 \%$ del conjunto.

\subsection{Preferencias de las profesoras: distribución por áreas}

Si cuando analizábamos la evolución de la proporción de mujeres en la universidad granadina observábamos una paralización en la equiparación entre el profesorado masculino y femenino en el conjunto del profesorado, tampoco se advierte un

${ }^{2}$ Los datos correspondientes a los años 1975-1980 han sido consultados en: I.N.E. Estadística de la Educación Superior en España (Madrid). Madrid y los de los años 1981-1990 en: Consejo de Universidades. Anuario de Estadística Universitaria (Madrid). 
mayor equilibrio al analizar la distribución por sexos entre las grandes áreas de conocimiento. La mayor presencia de mujeres se encuentra en CMV y CHS, con porcentajes de participación entre el 33.1\% y el 30.8\%, duplicándose la participación femenina de 1975 en 1990 en CH. En cambio, en CT la presencia femenina en 1975 es bastante similar a la que se daba en 1990, área en la que incluso la participación cae hasta un $20.2 \%$ (ver tab. 2).

Tabla 2

Porcentaje de mujeres en el profesorado universitario según grandes área de conocimiento

\begin{tabular}{rrrrrrrrrrr}
\hline & \multicolumn{3}{c}{ CHS } & \multicolumn{3}{c}{ CT } & \multicolumn{5}{c}{ CMV } \\
& $\begin{array}{c}\text { Ambos } \\
\text { sexos }\end{array}$ & Mujeres & $\%$ Muj & $\begin{array}{c}\text { Ambos } \\
\text { sexos }\end{array}$ & Mujeres & $\%$ Muj & $\begin{array}{c}\text { Ambos } \\
\text { sexos }\end{array}$ & Mujeres & $\%$ Muj \\
\hline 1975 & 47 & 7 & 14.9 & 49 & 10 & 20.4 & 48 & 10 & 20.8 \\
1976 & 62 & 11 & 17.7 & 59 & 11 & 18.6 & 55 & 11 & 20.0 \\
1977 & 71 & 12 & 16.9 & 62 & 10 & 16.2 & 59 & 12 & 20.4 \\
1978 & 91 & 16 & 17.6 & 74 & 15 & 20.3 & 69 & 12 & 17.4 \\
1979 & 103 & 19 & 18.5 & 81 & 15 & 18.5 & 82 & 19 & 23.2 \\
1980 & 115 & 20 & 17.4 & 88 & 18 & 20.5 & 94 & 22 & 23.4 \\
1981 & 147 & 30 & 20.4 & 103 & 20 & 19.4 & 104 & 25 & 24.1 \\
1982 & 152 & 34 & 22.4 & 117 & 22 & 18.8 & 118 & 30 & 25.4 \\
1983 & 196 & 38 & 19.4 & 163 & 27 & 16.6 & 152 & 40 & 26.3 \\
1984 & 238 & 52 & 21.8 & 211 & 34 & 16.1 & 179 & 47 & 26.3 \\
1985 & 356 & 109 & 30.6 & 253 & 42 & 16.6 & 228 & 64 & 28.1 \\
1986 & 427 & 124 & 29.1 & 312 & 47 & 15.1 & 279 & 82 & 29.4 \\
1987 & 590 & 175 & 29.6 & 426 & 76 & 17.8 & 411 & 135 & 32.8 \\
1988 & 768 & 237 & 30.9 & 527 & 102 & 19.4 & 472 & 156 & 33.1 \\
1989 & 1.023 & 322 & 31.5 & 715 & 137 & 19.2 & 570 & 195 & 34.2 \\
1990 & 1.253 & 386 & 30.8 & 893 & 180 & 20.2 & 659 & 218 & 33.1 \\
\hline
\end{tabular}

El crecimiento en cada una de las grandes áreas de conocimiento ha sido similar en CHS y CMV, mientras que en CT ha crecido de forma independiente, debido a que ha sido el área de enseñanza tradicionalmente más masculinizada. 
Feminización de la Universidad de Granada...

En CHS el porcentaje de profesoras con respecto al total del profesorado decrece y crece de forma paralela desde 1975 hasta 1990, el mayor crecimiento de profesorado tanto femenino como masculino se produce en 1985 (109.6\% y 32.8\%, respectivamente). El menor crecimiento en el profesorado femenino se da en 1980 (5.3\%) y en el masculino dos años más tarde (0.8\%). En cambio en CT se da el caso contrario, el profesorado masculino es el que decrece y crece a la par que el total del profesado, el mayor incremento del profesorado masculino se da en 1983 (43.3\%) mientras que en el profesorado femenino se da en 1987 (61.7\%), a partir de este año el número de profesoras crece y decrece en los mismos años que el profesorado masculino. El menor incremento entre profesorado femenino se da en 1977 (-9.1\%) siendo para el profesorado masculino en 1980 (6.1\%). En el área de CMV el crecimiento del profesorado femenino evoluciona de manera diferente al masculino, que lo hace a la vez que el total del profesorado, pero a partir de 1981 hasta 1990 aumentan y disminuyen en los mimos años teniendo el mayor crecimiento en 1987 con un $64.4 \%$ para el profesorado femenino y un 50.3\% para el masculino. El decrecimiento mayor para el profesorado femenino es en 1978 (0.0\%) y para el masculino es en 1977 (6.8\%) (tabla 3).

\section{Tabla 3}

Tasa anual de crecimiento del profesorado femenino y masculino por grandes áreas de conocimiento

\begin{tabular}{rrrrrrrrrr}
\hline & \multicolumn{3}{c}{ CHS } & \multicolumn{3}{c}{ CT } & \multicolumn{3}{c}{ CMV } \\
& \multicolumn{1}{c}{ Muj. } & Hom. & $\begin{array}{c}\text { Amb. } \\
\text { sexos }\end{array}$ & Muj. & Hom. & $\begin{array}{c}\text { Ambos } \\
\text { sexos }\end{array}$ & Muj. & Hom. & $\begin{array}{r}\text { Amb. } \\
\text { sexos }\end{array}$ \\
\hline 1975 & & & & & & & & & \\
1976 & $57,2 \%$ & $27,5 \%$ & $31,9 \%$ & $10,0 \%$ & $23,1 \%$ & $20,4 \%$ & $10,0 \%$ & $15,8 \%$ & $14,6 \%$ \\
1977 & $9,1 \%$ & $15,7 \%$ & $14,5 \%$ & $-9,1 \%$ & $8,3 \%$ & $5,1 \%$ & $9,1 \%$ & $6,8 \%$ & $7,3 \%$ \\
1978 & $33,3 \%$ & $27,1 \%$ & $28,2 \%$ & $50,0 \%$ & $13,5 \%$ & $19,4 \%$ & $0,0 \%$ & $21,3 \%$ & $16,9 \%$ \\
1979 & $18,7 \%$ & $12,0 \%$ & $13,2 \%$ & $0,0 \%$ & $11,9 \%$ & $9,5 \%$ & $58,3 \%$ & $10,5 \%$ & $18,8 \%$ \\
1980 & $5,3 \%$ & $13,1 \%$ & $11,6 \%$ & $20,0 \%$ & $6,1 \%$ & $8,6 \%$ & $15,8 \%$ & $14,3 \%$ & $14,6 \%$ \\
1981 & $50,0 \%$ & $23,2 \%$ & $27,8 \%$ & $11,1 \%$ & $18,6 \%$ & $17,0 \%$ & $13,6 \%$ & $9,7 \%$ & $10,6 \%$ \\
1982 & $13,3 \%$ & $0,8 \%$ & $3,4 \%$ & $10,0 \%$ & $14,5 \%$ & $13,6 \%$ & $20,0 \%$ & $11,4 \%$ & $13,5 \%$ \\
1983 & $11,7 \%$ & $33,9 \%$ & $28,9 \%$ & $22,7 \%$ & $43,2 \%$ & $39,3 \%$ & $33,3 \%$ & $27,3 \%$ & $28,8 \%$ \\
\hline
\end{tabular}




\begin{tabular}{lccccccccc}
\hline 1984 & $36,8 \%$ & $17,7 \%$ & $21,4 \%$ & $25,9 \%$ & $30,2 \%$ & $29,4 \%$ & $17,5 \%$ & $17,8 \%$ & $17,7 \%$ \\
1985 & $19,6 \%$ & $32,8 \%$ & $49,6 \%$ & $23,5 \%$ & $19,2 \%$ & $19,9 \%$ & $36,2 \%$ & $24,3 \%$ & $27,4 \%$ \\
1986 & $13,7 \%$ & $22,7 \%$ & $19,9 \%$ & $11,9 \%$ & $25,6 \%$ & $23,3 \%$ & $28,2 \%$ & $20,1 \%$ & $22,4 \%$ \\
1987 & $41,2 \%$ & $36,9 \%$ & $38,2 \%$ & $61,7 \%$ & $32,1 \%$ & $36,5 \%$ & $64,6 \%$ & $50,3 \%$ & $47,3 \%$ \\
1988 & $35,4 \%$ & $27,9 \%$ & $30,2 \%$ & $34,2 \%$ & $21,4 \%$ & $23,7 \%$ & $15,6 \%$ & $14,5 \%$ & $14,8 \%$ \\
1989 & $35,8 \%$ & $32,0 \%$ & $33,2 \%$ & $34,3 \%$ & $36,0 \%$ & $35,7 \%$ & $25,0 \%$ & $18,7 \%$ & $20,7 \%$ \\
1990 & $19,8 \%$ & $23,7 \%$ & $22,5 \%$ & $31,4 \%$ & $23,4 \%$ & $24,9 \%$ & $11,8 \%$ & $17,6 \%$ & $15,6 \%$ \\
\hline
\end{tabular}

Haciendo un estudio pormenorizado para cada una de las grandes áreas de conocimiento se encuentran áreas de enseñanza en las que durante éste período no ha existido presencia de mujeres en CHS, un $2.8 \%$ de un total 71 áreas. En CT la ausencia de profesoras ha sido aún mayor, un 34 \% de un total de 50 áreas, frente a CMV en las que las mujeres están representadas en todas las áreas.

La presencia femenina ha aumentado desde el año 1975 al 1990 en las distintas áreas de conocimiento pero en pocos casos conforman el 50\% del profesorado y en menor medida el $100 \%$. Si el incremento de profesoras ha sido progresivo y paulatino, su incorporación a nuevas áreas de enseñanza lo ha sido aún más (v. tab. 4).

\section{Tabla 4}

Presencia femenina en las áreas de enseñanza (1975-90)

\begin{tabular}{rrrrrrrrrrr}
\hline & \multicolumn{3}{c}{ CHS } & \multicolumn{4}{c}{ CT } & \multicolumn{4}{c}{ CMV } \\
& $\begin{array}{c}\text { Total- } \\
\text { áreas }\end{array}$ & $\begin{array}{c}\text { Presencia } \\
\text { Muj. }\end{array}$ & $\%$ Muj. & $\begin{array}{c}\text { Total- } \\
\text { áreas }\end{array}$ & $\begin{array}{l}\text { Presencia } \\
\text { Muj. }\end{array}$ & \%Muj. & $\begin{array}{c}\text { Total } \\
\text { áreas }\end{array}$ & $\begin{array}{c}\text { Presenc } \\
\text { Muj. }\end{array}$ & $\%$ \%uj. \\
\hline 1975 & 27 & 7 & 25.9 & 24 & 8 & 33.3 & 18 & 7 & 38.9 \\
1976 & 30 & 8 & 26.7 & 27 & 9 & 33.3 & 17 & 7 & 41.2 \\
1977 & 34 & 9 & 26.5 & 28 & 8 & 28.6 & 18 & 7 & 38.9 \\
1978 & 41 & 13 & 31.7 & 32 & 11 & 34.4 & 20 & 7 & 35.0 \\
1979 & 46 & 16 & 34.8 & 32 & 11 & 34.4 & 21 & 10 & 47.6 \\
1980 & 49 & 17 & 34.7 & 32 & 12 & 37.5 & 21 & 11 & 52.4 \\
1981 & 54 & 22 & 40.7 & 32 & 13 & 40.6 & 21 & 11 & 52.4 \\
1982 & 52 & 23 & 44.3 & 34 & 14 & 41.2 & 22 & 12 & 54.5 \\
1983 & 55 & 24 & 43.6 & 37 & 15 & 40.5 & 24 & 14 & 58.3 \\
1984 & 59 & 30 & 50.8 & 37 & 17 & 45.9 & 25 & 16 & 64.0 \\
\hline
\end{tabular}


Feminización de la Universidad de Granada...

\begin{tabular}{rrrrrrrrrr}
\hline 1985 & 64 & 42 & 65.6 & 39 & 20 & 51.3 & 25 & 16 & 64.0 \\
1986 & 66 & 44 & 66.7 & 40 & 22 & 55 & 25 & 18 & 72.0 \\
1987 & 67 & 49 & 73.2 & 42 & 23 & 54.7 & 25 & 22 & 88.0 \\
1988 & 69 & 59 & 85.5 & 44 & 27 & 61.4 & 25 & 22 & 88.0 \\
1989 & 71 & 68 & 95.7 & 48 & 30 & 62.5 & 25 & 23 & 92.0 \\
1990 & 71 & 69 & 97.2 & 50 & 33 & 66 & 25 & 25 & 100.0 \\
\hline
\end{tabular}

CHS en el año 1975 contaba con siete profesoras que ocupaban siete áreas de enseñanza, cinco de ellas en una proporción del 100\% con respecto al total de profesores en el área, las otras dos con una proporción del 50\% y del 33\%, para el año 1990 este porcentaje tan sólo se mantiene en Filología italiana. Las áreas en las que la presencia de profesoras es mayor o igual al 50\% en el año 1990 son: Arqueología (100\%), Filología catalana (100\%), Filología italiana (100\%), Trabajo social y servicios sociales $(100 \%)$, Didáctica de la expresión musical (84.6\%), Filología alemana (77.8\%), Didáctica de la lengua y la literatura (73.9\%), Comercialización de mercado (66.7\%), Estudios árabes e islámicos (50\%), Estudios hebreos y arameos (50\%), Lingüística general (66.7\%), Música (50\%), y Organización de empresas (50\%).

La presencia de profesoras que ejercían su docencia en CT en 1975 era de diez distribuidas en ocho áreas de enseñanza, tres de ellas en una proporción del 100\% con respecto al total de profesoras en esa área, dos con una proporción del 50\%, otras dos con un 33.3\% y un área con un 40\% de presencia. Para el año 1990 sólo superan el 50\% en el área de Farmacia y tecnología farmacéutica con un 57.7\%. En el caso de CMV en 1975, también había diez profesoras pero distribuidas en siete áreas de enseñanza alcanzando dos de ellas el $100 \%$ en relación con el total de áreas, una con el $66.7 \%$, tres con el 50\% y una con el 25\% de presencia. En el año 1990 superan el 50\% en las siguientes áreas: Nutrición y bromatología (73.3\%), Microbiología (58.8\%), Parasitología (52.9\%), Fisiología (52.4\%), Enfermería (51.1\%) y Farmacología (50\%).

A partir de los datos analizados se comprueba que CHS que en 1975 era la que menor número de profesoras contaba en sus áreas de enseñanza, 14.9\%, en 1990 es en la 
que ejercen su docencia mayor número de mujeres $30.8 \%$, por tanto se afirma la feminización de las áreas de enseñanza en CHS en la Universidad de Granada. También observamos que en CT y CMV que en el año 1975 tenían un porcentaje de $20.8 \%$ y 20.4\% respectivamente eran áreas que aparentemente podría haber una posible feminización, cuando analizamos su evolución observamos que en 1990 es el área de CMV la que continúa una feminización con un porcentaje del $33.1 \%$ en contraposición con CT en la que sus áreas en 1990 mantienen un porcentaje menor que en 1975, un $22.2 \%$ de profesoras, manteniéndose como el área "tradicionalmente masculinizada".

\subsection{Las profesoras universitarias y la jerarquía docente}

En 1980 más de las tres cuartas partes del profesorado universitario español era personal contratado en situación de precariedad. La aplicación de la L.R.U. en 1983 estabilizó a la mayoría de los Profesores No Numerarios (PNNs) a través del nuevo diseño que se hizo de los cuerpos docentes y de su sistema de acceso.

En la Universidad de Granada en el período 1975-1983, la proporción de profesoras con contratos de PNNs oscila entre el 25\% y el 32\% y el de funcionarias entre $8 \%$ y el $16 \%$, mientras que en el caso del profesorado masculino el porcentaje de PNNs varía entre el $67 \%$ y el $75 \%$ y el de funcionarios entre el $91 \%$ y el $83 \%$. A partir de la L.R.U., en el período 1984-1990, los cambios de la situación del profesorado no se reflejan hasta 1985, aunque el porcentaje de funcionarias oscila entre un $22 \%$ y un $26 \%$, el porcentaje de profesoras PNNs sigue en aumento entre un 29\% y un 37\%; en cambio, el porcentaje de profesores funcionarios continúa siendo mayor, oscila entre un $73 \%$ y $77 \%$ y el de PNNs entre un $62 \%$ y un $70 \%$ (v. tab. 5). 
Feminización de la Universidad de Granada...

Tabla 5

Distribución del profesorado en las categorías docentes de funcionario y asociado por $\operatorname{sexos}(1975-90)$

\begin{tabular}{rrrrrrrrrrrr}
\hline \multicolumn{3}{c}{ Funcionarios } & \multicolumn{1}{c}{ PNNs/Asociados } \\
\multicolumn{1}{r}{} & \multicolumn{1}{c}{ Total } & Muj. & \multicolumn{1}{c}{$\%$ M } & Hom. & \multicolumn{1}{c}{$\%$} & Total & Muj. & $\% M$ & Hom. & $\% H$ \\
\hline 1975 & 72 & 6 & 8.3 & 66 & 91.7 & 72 & 21 & 29.2 & 51 & 70.8 \\
1976 & 84 & 9 & 10.7 & 75 & 89.3 & 92 & 24 & 26.1 & 68 & 73.9 \\
1977 & 92 & 9 & 9.8 & 83 & 90.2 & 100 & 25 & 25.0 & 75 & 75.0 \\
1978 & 124 & 14 & 11.3 & 110 & 88.7 & 110 & 29 & 26.4 & 81 & 73.6 \\
1979 & 145 & 19 & 13.1 & 126 & 86.9 & 121 & 34 & 28.1 & 87 & 71.9 \\
1980 & 161 & 21 & 13.1 & 140 & 86.9 & 136 & 39 & 28.7 & 97 & 71.3 \\
1981 & 210 & 34 & 16.2 & 176 & 83.8 & 144 & 41 & 28.5 & 103 & 71.5 \\
1982 & 230 & 36 & 15.6 & 194 & 84.4 & 157 & 50 & 31.8 & 107 & 68.1 \\
\hline 1983 & 341 & 49 & 14.4 & 292 & 85.6 & 170 & 56 & 32.9 & 114 & 67.1 \\
\hline 1984 & 454 & 72 & 15.8 & 382 & 84.2 & 173 & 61 & 35.3 & 112 & 64.7 \\
1985 & 673 & 154 & 22.9 & 519 & 77.1 & 163 & 61 & 37.4 & 102 & 62.6 \\
1986 & 860 & 197 & 22.9 & 663 & 77.1 & 158 & 56 & 35.4 & 102 & 64.6 \\
1987 & 1118 & 287 & 25.7 & 831 & 74.3 & 309 & 99 & 32.1 & 210 & 67.9 \\
1988 & 1258 & 334 & 26.5 & 924 & 73.5 & 509 & 161 & 31.6 & 348 & 68.4 \\
1989 & 1414 & 375 & 26.5 & 1039 & 73.5 & 895 & 279 & 31.2 & 616 & 68.8 \\
1990 & 1617 & 429 & 26.5 & 1188 & 73.5 & 1188 & 355 & 29.9 & 833 & 70.1 \\
\hline
\end{tabular}

En ambos períodos la situación del profesorado con contratos PNNs varía muy poco, la proporción del primer período se mantiene en el segundo. Sin embargo, con respecto a la situación del personal funcionario, observamos un aumento considerable de funcionarias, mientras que el porcentaje de funcionarios diminuye. Aunque sigue habiendo un desequilibrio entre el profesado masculino y femenino, se puede afirmar que la situación de las profesoras al igual que los profesores fueron favorecidos por esta política, aumentando la situación funcionarial entre éstas en los últimos años. El análisis de las cifras anteriores indica que si el porcentaje de profesoras en el conjunto del profesorado es muy pequeño, un $27.0 \%$ en 1990 , esta proporción apenas crece entre el profesorado no numerario $29.9 \%$. 
La presencia femenina en las categorías de funcionario varía en función del nivel y tipo de enseñanza. En 1975 sólo el 14.3\% de los titulares eran mujeres, en 1990 la cifra llega al 28.4\%; en cambio las profesoras que imparten su docencia en Escuelas Universitarias presentan una situación más favorable, así en 1975 no había ninguna funcionaria sin tesis, en 1976 un $33.3 \%$ y en 1990 representaban el $34.7 \%$ de los funcionarios $\sin$ tesis. Conforme se asciende el escalafón profesional desciende considerablemente la presencia mujeres (BALLARÍN y MARTÍNEZ, 1989: 45), hasta el año 1981 no había ninguna catedrática, y su proporción era de un $1.6 \%$ del total de catedráticos y en 1990 de un $6.9 \%{ }^{3}$.

Estas proporciones varían cuando se hace un análisis por áreas de conocimiento. En cuanto al profesorado funcionario, el área que tiene la proporción más alta de catedráticas para el año 1990 es la de CMV (14.3\%), le sigue el área de CT (4.9\%), y por último CHS (3.3\%). Como era de esperar la distribución de titulares mantiene el mismo orden de proporción que de catedráticas, en 1990 el porcentaje más alto es el del área de CMV (33.6\%), seguida de las CHS (28.4\%) y de CT (23.9\%), pero en esta categoría en valores absolutos el número más alto de profesoras titulares está en el área de CHS. El grupo de los funcionarios sin tesis para el año 1990 sigue el mismo orden en CMV (62.1\%), CHS (41.9\%) y CT (10.9\%), en valores absolutos el área de CHS es la que tiene un número mayor de profesoras.

En cuanto al profesorado contratado, el área en la que hay más mujeres PNNs a tiempo completo y con grado de doctor en el año 1990 es CMV (45.3\%), seguida de CT

\footnotetext{
${ }^{3}$ Puede consultarse la correspondiente tabla de distribución del profesorado por años y categorías académicas en Presencia y producción científica de las profesoras en la Universidad de Granada (1975-1990), actualmente en prensa (Muñoz Muñoz, 2006). Ana Ma . Granada: Universidad de Granada, Servicio de Publicaciones, 2006 (En prensa).
} 
Feminización de la Universidad de Granada...

(40.4\%) y de CHS (22.6\%). Con PNNs no doctores el área que tiene una proporción más alta es CHS (43.9\%), seguida de CMV (38.1\%) y de CT (25.6\%). Con PNNs a tiempo parcial CMV tiene la proporción más alta de mujeres (28.9\%), seguida de CHS (11.0\%) y de CT (6.7\%).

Haciendo un balance de los datos expuestos anteriormente se confirma que la presencia de mujeres con respecto al total del profesorado en categorías de contratación es más alta y en especial cuando son doctoras, en el caso de las áreas de CT y de CMV, en CHS la presencia es más alta en la categoría de PNNs no doctores. También se comprueba que la incorporación de mujeres en la categoría más alta es muy tardía con respecto a la de los varones, mientras que ellos se incorporan desde el principio del período estudiado y con proporciones muy elevadas, las mujeres se incorporan más tarde, y en años distintos dependiendo de las áreas, así las primeras catedráticas en CT se incorporan en el año 1981, seguida de CMV en 1983 y por último CHS en 1986, sería lógico suponer que las áreas con mayor número de mujeres tendrán mayor número de catedráticas, y sin embargo esta correspondencia no se da en algunos casos (véase GARCÍA, M, y GARCÍA, M.A., 1997). Así, se comprueba que en 1990 CHS tiene un total de 386 de mujeres que conforman el área y el número de catedráticas es de 2; en CT con 180 mujeres tan sólo hay 3; y en CMV con un total de 218 mujeres el número de catedráticas es el más elevado de 7 (un $14.3 \%$ de 49 catedráticos). Otra de las categorías en la que la presencia de mujeres es tardía es la de funcionarias sin tesis en las áreas de CT y CMV, pero este dato es poco significativo pues se debe a la creación de nuevas Escuelas Universitarias.

\section{Conclusiones}

La presencia de las mujeres en el sistema educativo universitario es cada vez mayor. A lo largo de los dieciséis años estudiados, 1975-1990, aumenta el profesorado femenino en la Universidad de Granada, fruto tanto del incremento total del profesorado, como de la mayor presencia de mujeres que lo conforma. Este crecimiento ha sido constante y se manifiesta en la incorporación de las profesoras a nuevas áreas de conocimiento en las que anteriormente sólo ejercían varones. Con la L.R.U. el 
profesorado, tanto el femenino como el masculino, se benefició en la estabilización laboral. Pero a partir de la Reforma sólo una minoría de profesoras ha llegado a la categoría académica más alta, la de catedrático/a. La evolución del número de profesoras ha sido positiva, aunque podría ser vista como negativa al producirse un estancamiento en la equiparación entre mujeres y hombres en el conjunto del profesorado.

El profesorado femenino en las grandes áreas de conocimiento es siempre inferior al masculino, siendo CMV y CHS las que cuentan con el mayor número de profesoras y $\mathrm{CT}$ con el menor. El número de profesoras funcionarias, al igual que el de profesores, se incrementó en los últimos años del período de estudio, favorecido por la L.R.U. No obstante continúa existiendo un desequilibrio entre el funcionariado femenino en favor del masculino. La incorporación de las mujeres a la categoría más alta es mucho menor y más tardía con respecto a los varones. Mientras que ellos se incorporan desde el principio del período que estudiamos, las primeras catedráticas aparecen en el año 1981 en CT, y en 1983 en CMV. 
Feminización de la Universidad de Granada...

\section{Bibliografía}

ALBERDI, Inés y ALBERDI, Isabel (1984): “Mujer y Educación: un largo camino hacia la igualdad de oportunidades". En Revista de Educación, n 275, p. 10.

ARENAL, Concepción (1989): La mujer del porvenir. La mujer de su casa. Barcelona: Orbis.

Ballarín Domingo, Pilar y Martínez LóPez, Cándida (1989): "La Universidad, un espacio también de, para, por, según... las mujeres”. En Primeras Jornadas de Educación no Sexista de Castilla-La Mancha. Villarobledo (Albacete): Dirección General de la Mujer de Castilla-La Macha, pp. 42-49.

Campo Alange, Condesa de (1963): Evolución de los métodos pedagógicos (En torno a María de Maeztu). La mujer en España: cien años de su historia: 1860-1960. Madrid: Aguilar.

CAPEl MARTínez, Rosa M. (1986): El trabajo y la educación de la mujer en España (1900-1930). Madrid: Ministerio de Cultura, Instituto de la Mujer.

Consejo de Universidades (ed.) (1988-): Anuario de Estadística Universitaria (varios años). Madrid: Consejo de Universidades. Ministerio de Educación y Ciencia. Nota: Recoge los datos a partir del año 1981.

DURÁN HERAS, M. Ángeles (1981): La investigación sobre la mujer en la Universidad española contemporánea. Madrid: Ministerio de Cultura.

FLECHA GARCÍA, Consuelo (1996) Las primeras universitarias en España, 1872-1910. Madrid: Narcea.

García de Cortazar, Marisa y García de LEón, María Antonia (1997): Mujeres en minoría: Una investigación sociológica sobre las catedráticas de universidad en España. Madrid: Centro de Investigaciones Sociológicas.

INE (ed.) Estadística de la Enseñanza Superior en España (varios años). Madrid: Instituto Nacional de Estadística. 
176

Ana María Muñoz-Muñoz

MuÑoz MuÑoz, Ana M. (2006): Presencia y producción científica de las profesoras en la Universidad de Granada (1975-1990). Granada: Universidad de Granada, Servicio de Publicaciones (en prensa).

TORRES RAMÍREZ, Isabel de (2001): "Mujeres en la Universidad de Granada. Un camino aún por recorrer”. En Ruiz Rodríguez, Antonio Ángel. Memoria Gráfica de la Universidad de Granada: Archivos fotográficos. Granada: Universidad de Granada, pp. 180-188. 